\title{
Applying Virtual Reality Techniques to the Interactive Stress Analysis of a Tractor Lift Arm
}

\author{
By \\ Michael J. Ryken \\ Dr. Judy M. Vance \\ Iowa Center for Emerging Manufacturing Technology \\ Iowa State University \\ Ames, Iowa
}

\begin{abstract}
The objective of this research is to examine the challenges of applying virtual reality techniques to the interactive stress analysis of a tractor lift arm. NURBS-based free form deformation, finite element analysis, sensitivity analysis, collision detection, and virtual reality are combined to create an interactive environment for designers to view and modify part shape, evaluate the resulting stresses, and check for interference of the new part shape and surrounding parts in real-time. These methods are implemented using a surround screen virtual environment where the part of interest and associated geometry surrounding that part are displayed together using stereo projection to provide a three-dimensional view of the assembly.
\end{abstract}




\section{Introduction}

Manufacturing companies are continually trying to reduce the time required to take an idea from product conception to market so they can increase their competitiveness, maintain or increase their market share, and ultimately gain higher profits. This time reduction means fewer hours to develop the product, and fewer physical prototypes of the design that can be tested. With developments in computer codes, designers now rely more heavily on computer analysis and simulation as a way to reduce the number of costly prototypes and the time required to test them. Analysis and simulation leads to fewer prototypes, because it allows designers to solve many design problems before the first prototypes are built. Finite element analysis (FEA) is one form of structural analysis commonly used by manufacturing companies.

Virtual reality (VR) is a unique human-computer interface that allows the user to use natural head and hand movements to interact with the objects in the environment. Companies like Boeing[10], Volkswagen[1], Chrysler[7], Ford[2], Caterpillar[6], General Motors[4] and others are evaluating the use of VR techniques to reduce the number of physical prototypes by evaluating designs using a virtual environment. Since only computer models are evaluated, many virtual prototypes can be created and evaluated before the first physical prototypes are built. This saves the company time and money and helps them produce a better end product than was possible with traditional design methods.

The work presented here follows from methods developed by Yeh and Vance[12][13] where virtual reality techniques are used to provide the human-computer interface for analyzing FEA results. Yeh and Vance developed a method that allows designers to interactively change the shape of a part and view the effect the change has on the stresses in the part. Finite element analysis, Non-Uniform Rational B-Spline (NURBS)-based free form deformation, sensitivity analysis, design optimization, and virtual reality are combined to create an interactive environment for designers to view and modify part shape and evaluate the resulting stresses in a real-time three-dimensional virtual environment.

Unlike traditional structural analysis that is computationally intensive, interactive structural analysis uses shape sensitivities to approximate the stresses that result from the geometry manipulation. Implementing this method in a virtual environment enables the designer to use natural head and hand movements to examine the computer generated part, modify its shape, and evaluate the stresses that result from the change in real-time.

This research examines the challenges of applying this method to an industrial problem. The methods presented here extend previous work to include display and interaction in a surround screen virtual environment. Surrounding geometry and collision detection are also added to the environment to provide more information to the designer about the quality of the new design.

\section{Surround Screen Virtual Environment}

The surround screen virtual environment used in this research is the C2 at Iowa State University (Figure 1). The $\mathrm{C} 2$ is a second generation CAVE-like device. Computer images are projected on the front, left, and right walls, and the floor using BARCO 1208s projectors. These images are projected in stereo, where a separate computer image is created for the right eye and the left eye. Shutter glasses from StereoGraphics are worn 
by the user and synchronized to the computer graphics refresh rate by an infrared signal. The glasses alternately shutter between the left and right eye corresponding to the computer display of the left and right eye image. The images are refreshed at $96 \mathrm{~Hz}$, so the right image and left image are drawn 48 times each second. The brain does not have the ability to detect changes at this high frequency therefore, the two images are combined by the user's brain, and the objects drawn in the environment appear threedimensional.

The user's head and hand positions are tracked using an Ascension Flock of Birds magnetic tracker. The transmitter for the tracking system is suspended at the middle of the $\mathrm{C} 2$, and receivers are placed on one pair of CrystalEyes glasses and on an input device for one hand. Each tracking receiver continuously reports it's position and orientation ( 6 degrees of freedom) within the $\mathrm{C} 2$ relative to the transmitter. The receiver for the head position provides the computer with information about the user's viewpoint and orientation so that images are displayed based on where the user is standing and looking in the $\mathrm{C} 2$. The receiver on the hand gives the computer information about the hand location and orientation in the environment.

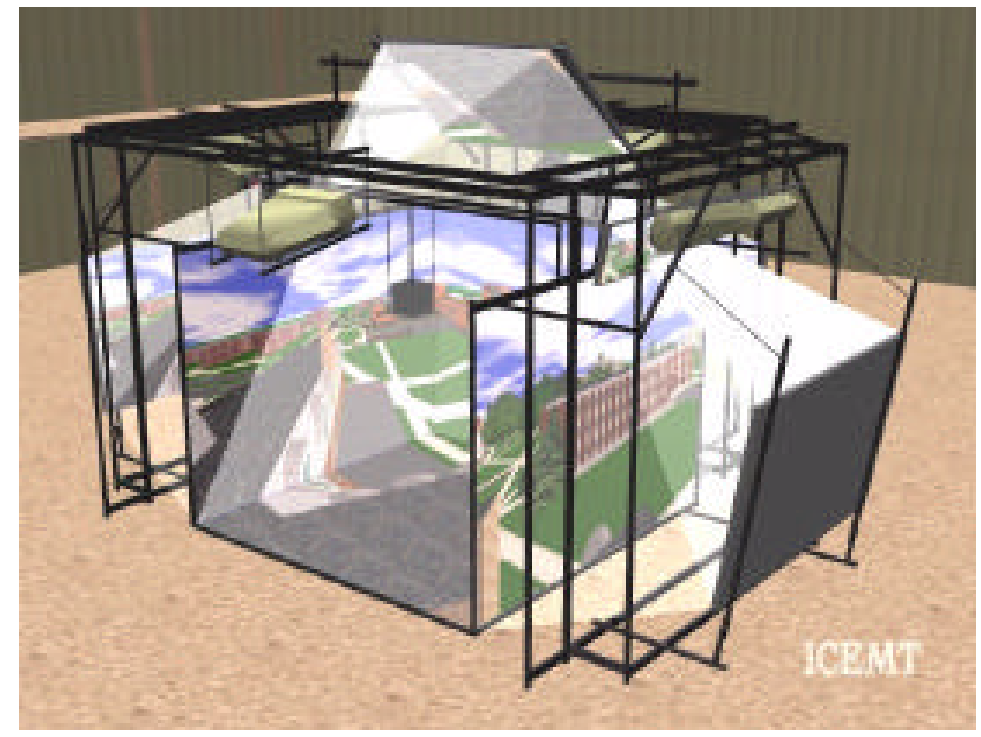

Figure 1. The C2 at Iowa State University

Three types of input devices are used in the C2: the wand, the Virtual Technologies Cyberglove, and the Fakespace Pinch Gloves. The wand consists of a commercial joystick with the base removed, and the input buttons connected to an Immersion Corporation Ibox. The Ibox uses serial communication to report each button's state (on/off). The Cyberglove consists of a glove with strain gages running along the tops of each finger. These strain gages measure finger bending. Based on the finger orientations, gestures can be programmed to control the virtual environment. The Pinch Gloves record finger contact only. Each finger tip and the palm of the glove contain electrical contact patches. When the contact patches touch, a circuit is completed and this information is used to control the virtual environment. The software used in the $\mathrm{C} 2$ consists of the $\mathrm{C} 2$ library which contains a set of $\mathrm{C}$ and $\mathrm{C}++$ functions that handle the viewing projections, and manage the hardware devices. 


\section{Problem Definition}

Working with the John Deere Product Engineering Center (PEC) in Waterloo, Iowa, a suitable design problem was identified. High stress had been observed in the right lift arm (Figure 2) of the three point hitch assembly on the 8000 Series tractor due to high vertical forces. These stresses only occurred in the right lift arm because the load that caused the stresses was offset to the right of the center of the tractor. This problem was selected because the stresses could be analyzed using finite element analysis and because a shape change in the area around the hub (area of high stress) could possibly interfere with the lift cylinder rod.

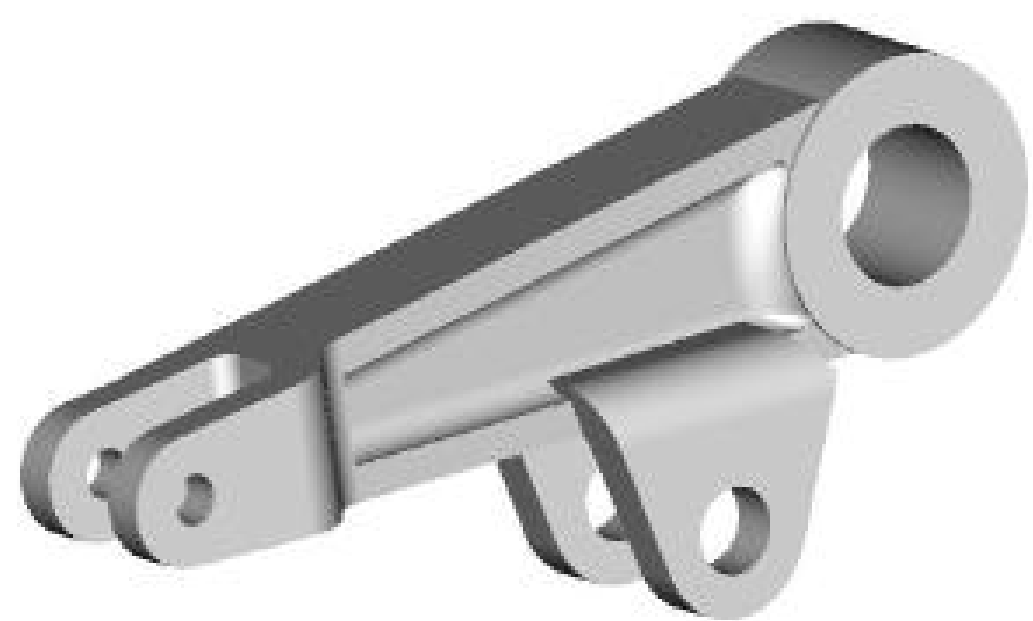

Figure 2. Lift arm from the John Deere 8000 series tractor

The lift arms are located on the rear of the tractor. They are cast parts and are the input links in a four bar mechanism that is used to raise and lower equipment that is mounted to the hitch. The right and left lift arms are connected to each other via the rockshaft. There are two hydraulic cylinders, one connected to each lift arm, that apply the force to raise and lower the arms. Lift links connect the lift arms to the draft links where the implement is mounted (Figure 3).

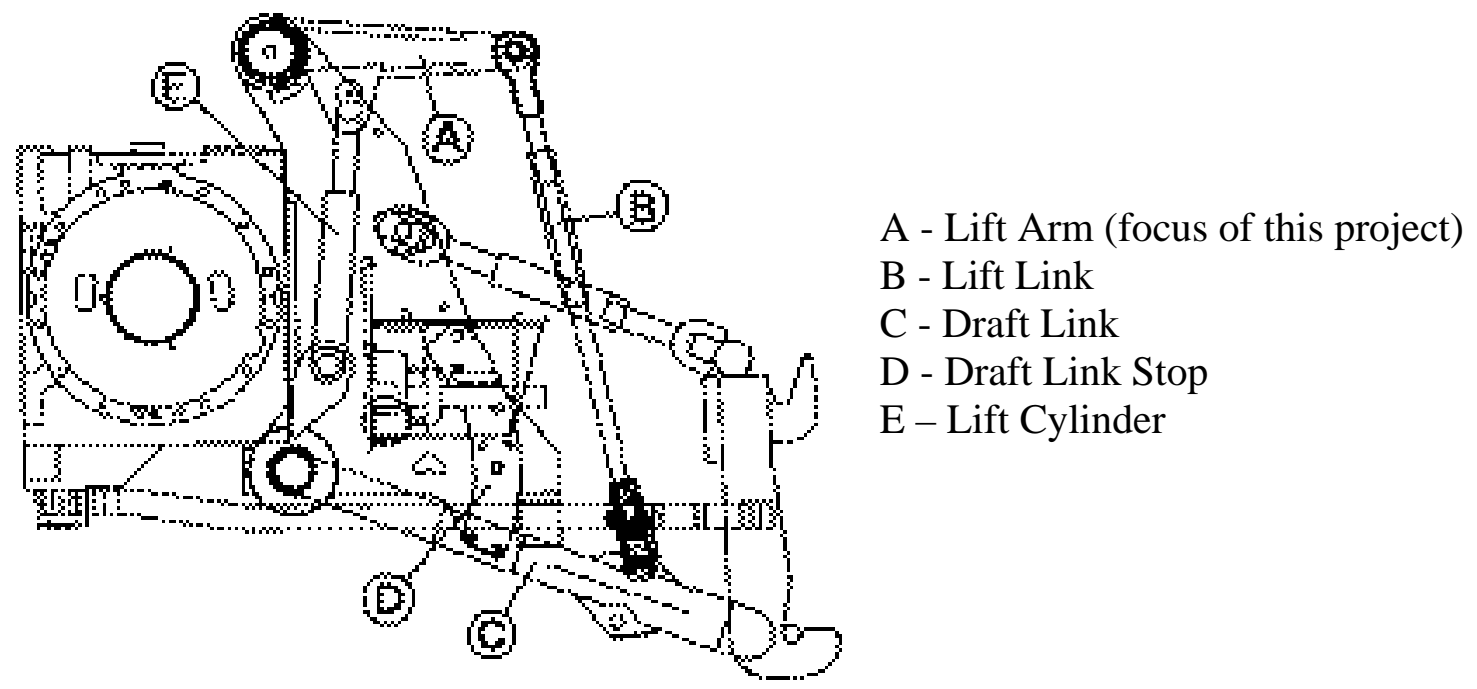

Figure 3. Side view of the hitch 


\section{Program Operation}

When the interactive stress analysis program is run, it loads information about the analysis part, the surrounding geometry, and the rest of the environment and displays it on the four surfaces of the C2 (Figure 4). The designer interacts with the three point hitch assembly using the wand to control a virtual hand. The buttons on the wand are used to create gestures with the virtual hand to control the program.

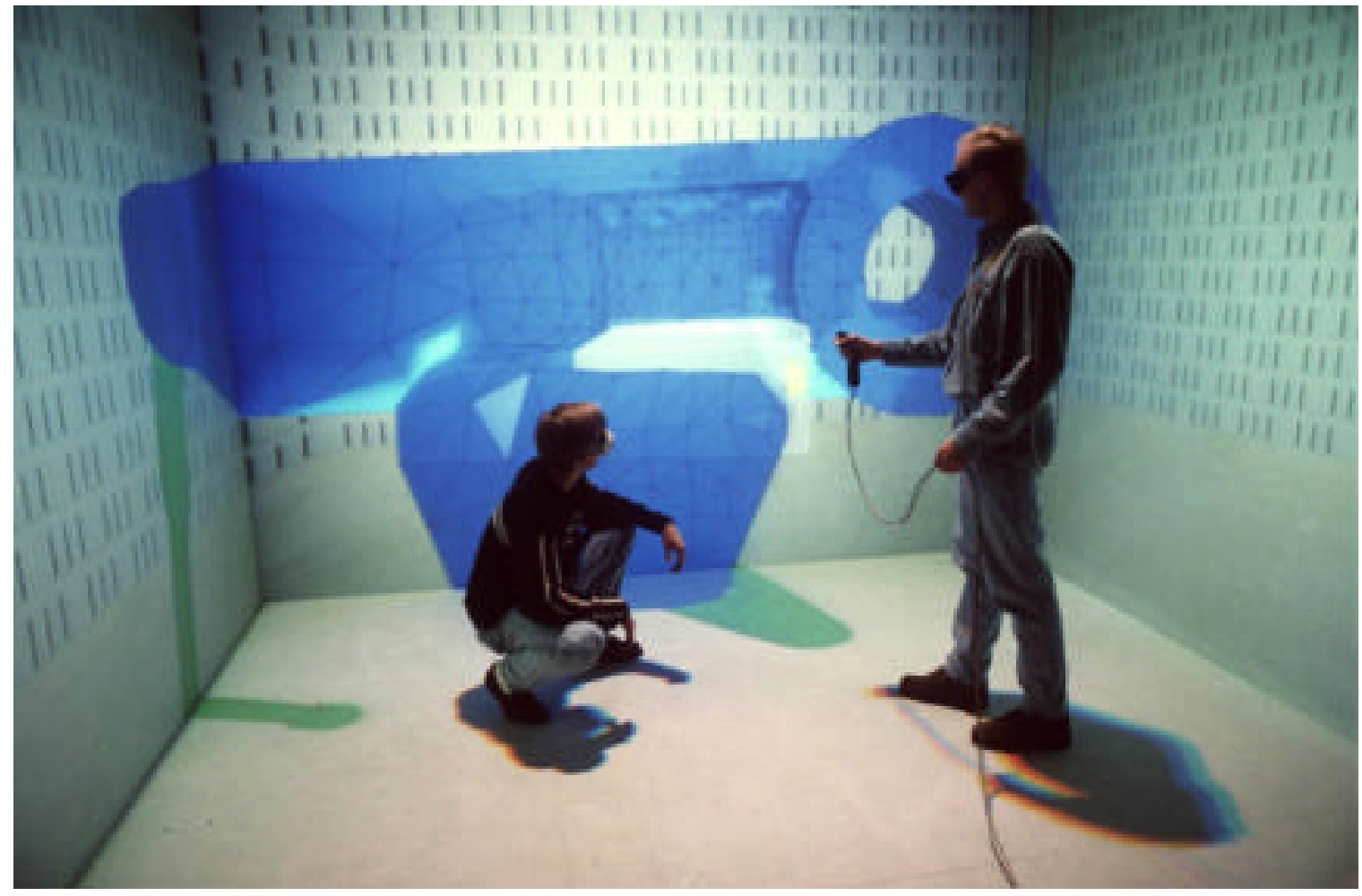

Figure 4. Interactive stress analysis program in C2

Because the sensitivity information can not be calculated in real-time, the operation of the program is divided into three parts. Figure 5 shows the three parts of the process, and the steps involved in each part. In this first part of the process, the design area is defined, the design variable is selected, and the MSC/NASTRAN[8] data file for the sensitivity analysis is created. The second part of the process involves calculating the sensitivities based on the design area and design variable selected in the first part. The final part of the process includes modifying the part shape to reduce the stresses in the part and checking for collisions between the new part shape and the surrounding geometry.

The program reads two input files, the initial FEA model and results, and surrounding geometry when it is started. The FEA model is displayed with the nodes in their displaced positions along with a color contour map of the stresses. A color key of the stresses is displayed so the designer knows the range of stresses in the part. The surrounding parts are displayed in their initial positions. The geometry is displayed in a texture mapped room to give the user the sense of being in a testing laboratory.

A menu (Figure 6) can be displayed in the environment from which the user can select operations to perform in the program. The virtual hand is displayed so the designer 


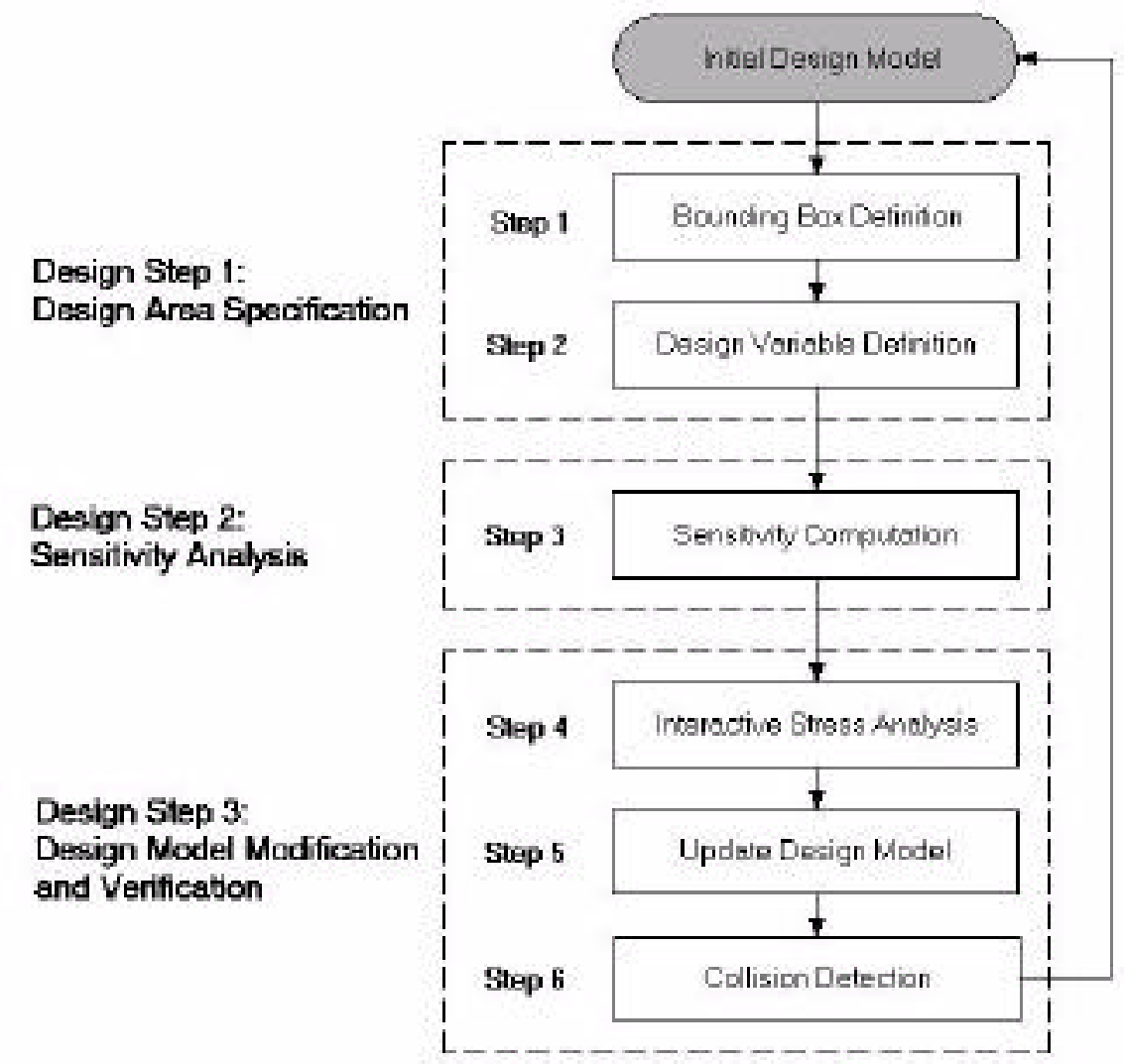

Figure 5. Interactive stress analysis steps

knows where he/she is pointing in the environment. While the menu is displayed, a ray extends in front of the virtual hand (Figure 6) for selecting options on the menu.

Using the menu, the designer can perform actions such as increasing or decreasing the size of the model using "Scale UP" and "Scale DOWN," animating the three point hitch mechanism up and down using "Animate UP" and "Animate DOWN," and changing the surrounding geometry from shaded to wire frame with the "Shaded"

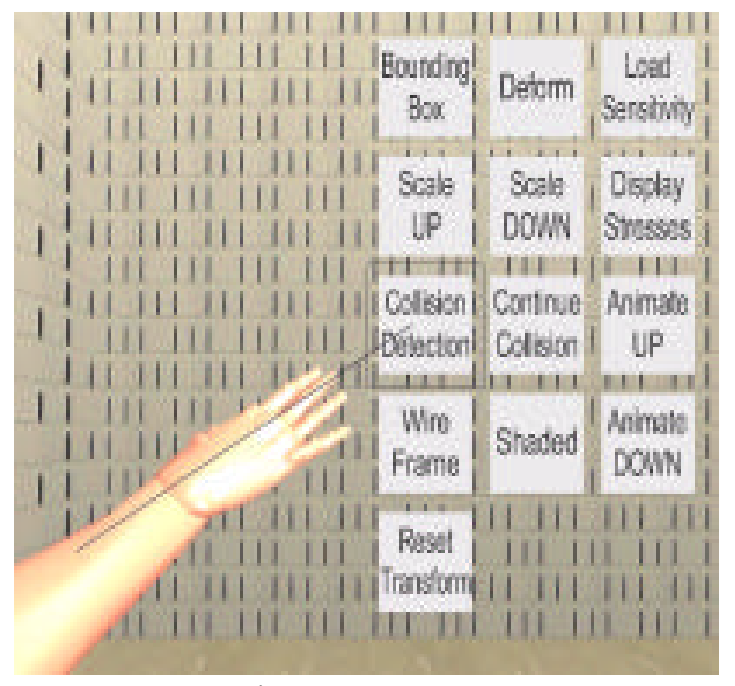

Figure 6. Menu 
and "Wire Frame" buttons. Other menu options are discussed later in this section.

The designer can also "grab" and move the geometry in the environment to examine it from different positions. The designer can move the assembly to any position and orientation in the environment. To place the assembly back into its original position, the designer selects the "Reset Transform" button on the menu.

The interactive stress analysis is performed as follows:

Step 1: Bounding Box Definition

The "Bounding Box" menu button is selected to define the design area. This displays a white semi-transparent regularly shaped 3D bounding box, similar to the "silk cursor"[14], that encloses the FEA model. The designer changes the size of this bounding box to enclose the area of the FEA model that will be reshaped. This is implemented to allow the designer to specify a particular region to reshape and also to preserve areas which should retain their original shape because of assembly considerations, etc.

Step 2: Design Variable Definition

When the design area is defined, the "Deform" button is selected from the menu. The design area is mapped to a NURBS volume, and the control point lattice replaces the semi-transparent bounding box. This method was developed in order to allow the designer to make smooth geometry changes to the finite element model. Locations of each FEA node are mapped to locations in the 3D NURBS volume which is defined by control points. Selecting and moving control points effectively causes changes in the nodal locations of each element. In this step, the designer selects certain control points of the NURBS volume to define the design variable. The design variable determines how the shape of the design area will change.

Step 3: Sensitivity Computation

With the design area and the design variable selected, a MSC/NASTRAN data file for the sensitivity analysis is created. The file that is created is similar to the original MSC/NASTRAN data file except that SOL 200 (Design Optimization) is used instead of SOL 101 (Statics) solution sequence. This file also contains the information about the design variable, the responses, and the design velocity field. In addition to creating the MSC/NASTRAN data file, the program also creates an ASCII file that contains information about the design area and the design variable.

The VR program is stopped and the sensitivity information is calculated using MSC/NASTRAN. When the sensitivity analysis is completed, the VR program is restarted. In the next part of the process, the sensitivity information is used to perform the interactive stress analysis, and the new part shape is checked for collisions with the surrounding geometry.

Step 4: Interactive Stress Analysis

After the program is restarted, the FEA model, surrounding geometry, and room are displayed as in the first part. The "Load Sensitivity" button is selected from the menu. The program then reads in the sensitivity information as well as the design area and design variable information that was saved in Step 3.

The designer now begins to change the shape of the FEA model and view the updated stresses interactively. The part shape changes according to the movement of the users hand. The new stresses are approximated using a linear Taylor series based on the sensitivity information. 


\section{Step 5: Update Design Model}

When the interactive stress analysis is complete, the updated node locations and stresses are stored in the VR program. The updated node information can be exported from the program to a MSC/NASTRAN data file. This file is analyzed with the finite element software to compare the actual solution with the approximate solution found using the interactive stress analysis program.

Step 6: Collision Detection

When the part shape has been changed to produce acceptable stresses in the part, collision detection is performed by selecting the "Collision Detection" button from the menu. The RAPID[3] collision detection algorithm is used to test for interference. To indicate collision detection mode, the FEA model color changes from the stress contour colors to white with red lines to show the boundary elements. Collisions are shown in red when parts collide. As soon as a collision is detected, the motion stops but can be resumed by selecting the "Continue Collision" button to check for collisions through the full range of motion of the lift arm mechanism. When collision checking is complete, the designer can view the stresses again by picking the "Display Stresses" button on the menu.

If the new shape of the lift arm collides with the surrounding geometry, the designer goes back to the interactive stress analysis, and changes the shape of the part again in an attempt to eliminate this collision. The designer may have to change the design area, and the design variable and perform the sensitivity analysis again to find an acceptable shape that does not interfere with other parts in the assembly.

\section{Analysis}

Engineers from John Deere measured the input loads from field testing under appropriate working conditions. The lift cylinder pressure, lift link force and lift arm angle were recorded. From this information, the largest lift link force was determined, and the corresponding lift arm angle and cylinder pressure were used to determine the input loads for the finite element analysis of the lift arm.

Important considerations for the finite element analysis in this application are that the mesh accurately models the part, and that it is not too large. The mesh should accurately model the part to obtain accurate results from the analysis in the area of interest. The mesh should also be small enough to maintain real-time interaction with the part during the interactive analysis. Because of the limitations of the rendering speed of the computer, the number of elements should be as small as possible. If the number of elements is too large, the computer can not render them fast enough to maintain real-time interaction with the lift arm during the interactive analysis.

A drawing of the lift arm was obtained from John Deere and modeled using Pro/Engineer[9]. Only the right arm was modeled and analyzed because the high stresses occurred in that arm. The arm was meshed using the automatic mesh generator in Pro/Engineer with tetrahedral elements. Initially, this mesh created a large number of elements that did not allow real-time interaction during the interactive stress analysis.

In order to gain real-time performance while maintaining accuracy in the area of high stress, the number of elements had to be reduced. This was accomplished by simplifying the geometric model in areas which were not of interest in this problem. The model was simplified using Pro/Engineer. Figure 7 shows the lift arm before and after 


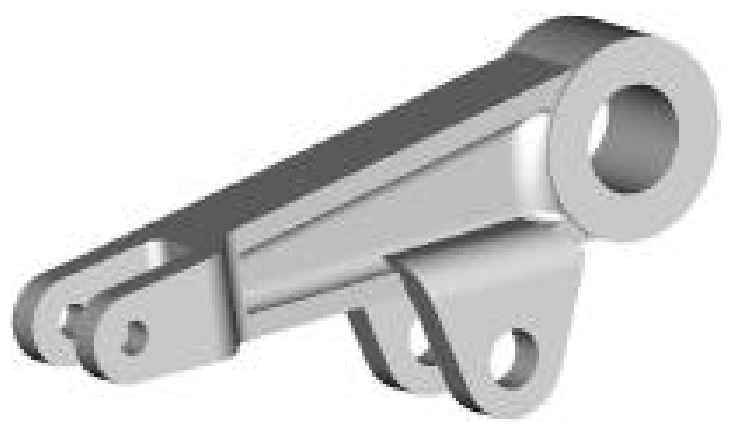

a.

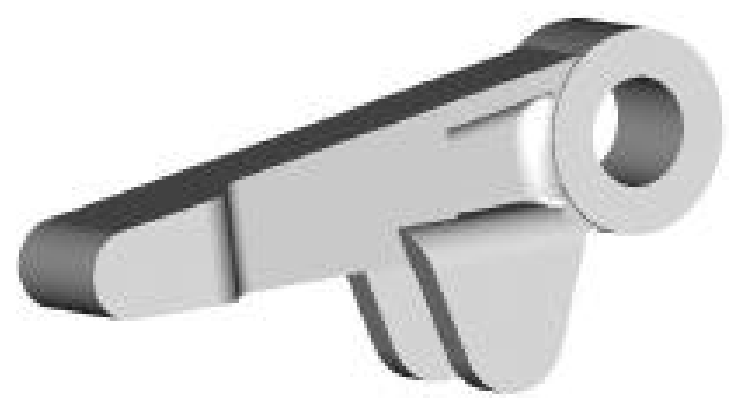

b.

Figure 7. Lift arm geometry (a) before and (b) after simplification

simplification.

The preprocessing steps for the model were somewhat lengthy, but eventually resulted in an acceptable analysis model. The final procedure involved importing the file created by Pro/Engineer into SDRC I-deas[11] where restraints that fixed the three translation directions were added to the nodes on the inside of the hub. These restraints were chosen because the right and left lift arms are attached through the rockshaft, so translation is restricted. The data file exported from I-deas was used in MSC/NASTRAN to perform the finite element calculations.

In order to validate the finite element model, a convergence study was performed. Convergence testing is used to calculate discretization error by increasing the number of degrees of freedom (DOF) by refining the mesh[5]. If the data of interest, in this case stress, does not approach a limiting value either the mesh is still too large, or the model is improperly defined.

The initial convergence test was performed with 22 analyses, each with increasing degrees of freedom. Concerns about the accuracy of the model in the area of interest around the hub led to adding a fillet from the outside of the arm to the cylinder yoke (Figure 8) and adding local mesh control in this area. This lead to an acceptable model for accuracy in the area of interest and size for real-time interaction. The final finite element model contains 2642 nodes and 11249 elements.

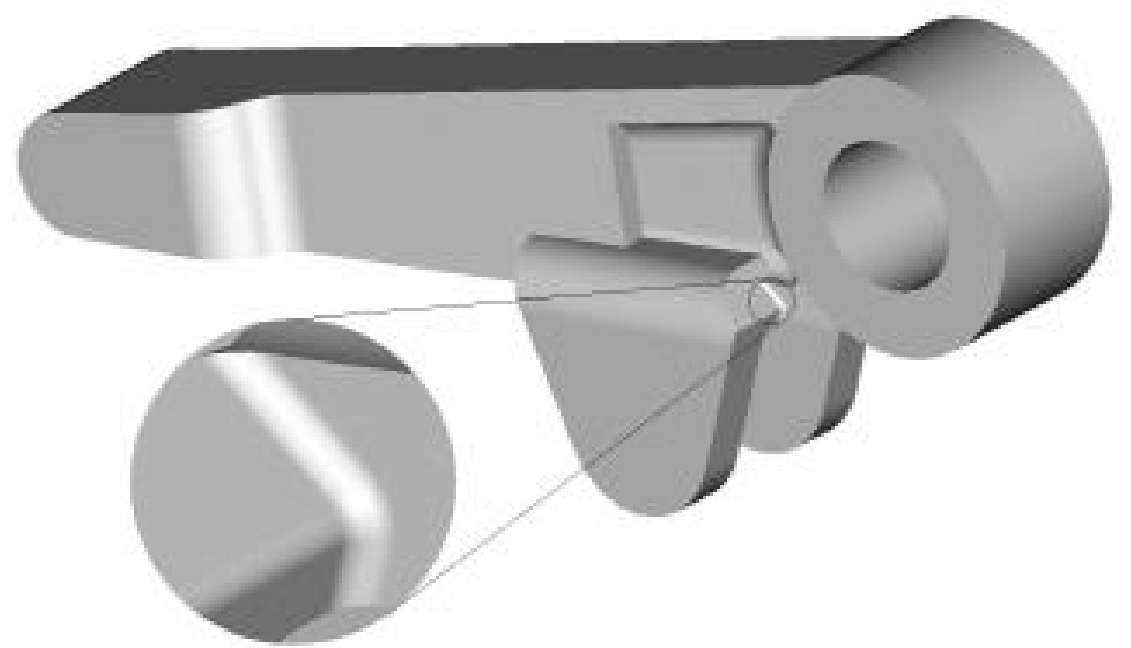

Figure 8. Fillet on lift cylinder yoke 


\section{Results}

In the first four solution iterations, the design area enclosed the fillet where the high stresses occurred (Figure 9). In each iteration, the selected design area was reduced to enclose less of the fillet each time. The first iteration enclosed the entire fillet and the fourth iteration enclosed only the area of the highest stress in the fillet. Each iteration contained three control points in one direction and two control points in the other two directions of the NURBS volume. Two control points were selected to modify the design area shape that were opposite the fillet. Figure 9 shows the fillet on the lift arm, the selected design area for each of the four iterations, and the selected control points.

While analyzing each of these iterations using the interactive stress analysis program it was found that the design area could not be modified enough to sufficiently reduce the stress and maintain a smooth shape. If the shape was changed enough to achieve sufficient stress reduction in the fillet area, sharp edges were produced on adjacent elements at the edges of the bounding box. These edges would cause high stress concentrations when the new shape of the part was reanalyzed. The solutions found from these design iterations were not acceptable.

The next approach was to select a design area on the bottom of the lift arm. This design area started near the hub and continued inside the lift cylinder yoke to the center of the lift cylinder attachment point. To prevent sharp edges from occurring on the

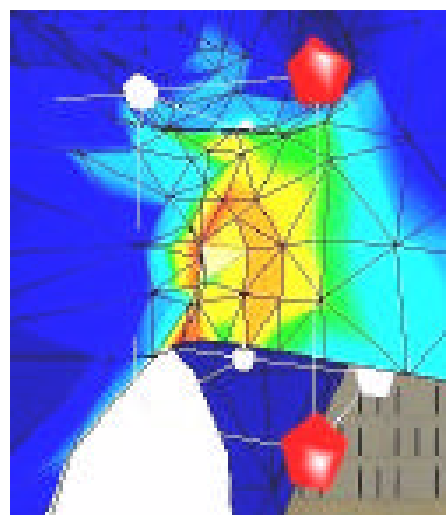

a.

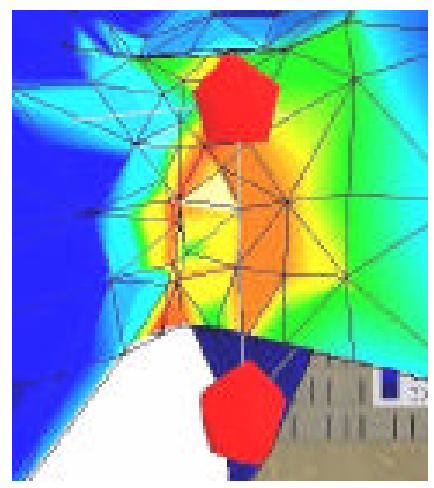

c.

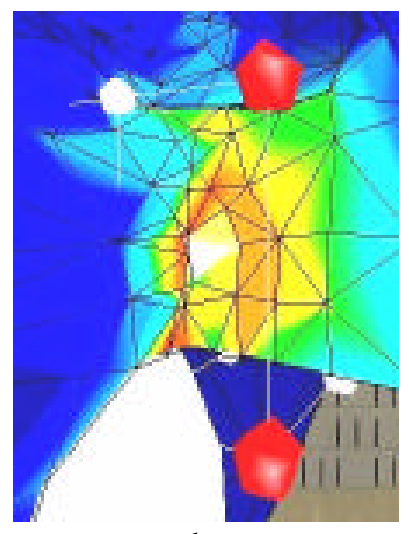

b.

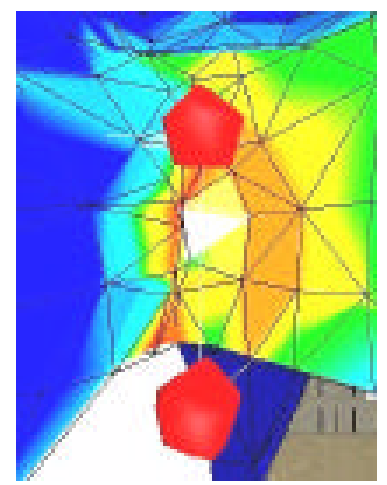

d.

Figure 9. Design area and design variable for the solution iterations (a) 1, (b) 2, (c) 3, and (d) 4. The selected control points are highlighted. 
bounding box edges, more control points were added to the NURBS volume. This iteration contained four control points in two directions and three in the third direction. The design area was selected with at least one element between the fillets on the inside of the lift cylinder yoke and the NURBS volume. The four center control points on the bottom of the design area were selected as the design variable in an attempt to maintain smoothness at the bounding box edges. Figure 10 is a view of the underside of the lift arm for this iteration showing the design area and the design variable.

The design variable was moved downward and the stress was reduced in the fillet area to an acceptable level and the elements remained smooth across the design area boundary. The mesh information for the new shape was saved to a MSC/NASTRAN data file for reanalysis. Errors during the analysis indicated that the aspect ratio of some elements was too large to solve the mesh. Because of these errors, the new shape could not be checked for correlation with the approximate solution from the interactive stress analysis program using data directly from the VR program.

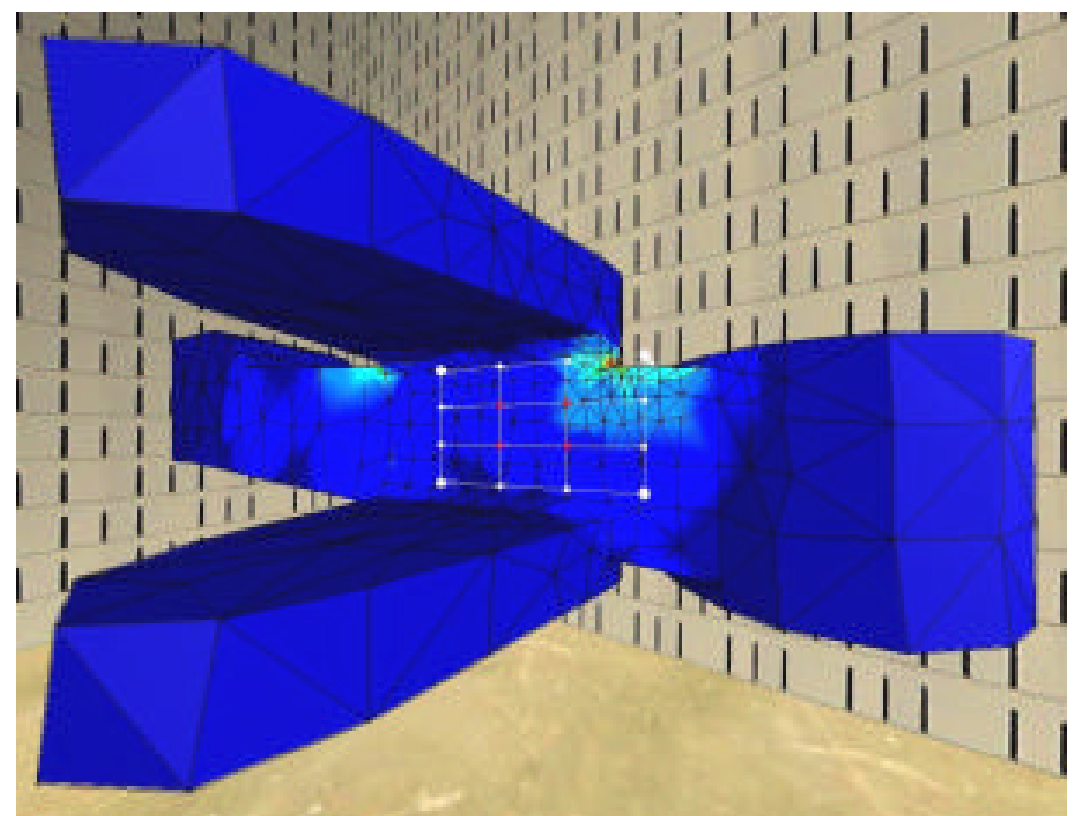

Figure 10. Design area and design variable for the fifth solution iteration

In the final iteration, the design area was selected similar to that shown in Figure 10 with one end of the design area located near the lift arm hub and the other end located near the center of the lift cylinder attachment point. This design area was wider than in the previous iteration in an attempt to produce elements that were not highly distorted. The NURBS volume contained four control points in two directions and three in the third direction. The six control points in the center of the bottom of the design area were selected as the design variable (Figure 11).

The design variable was moved downward $44 \mathrm{~mm}$ and the stresses were sufficiently reduced in the fillet area. Adjacent elements across the bounding box edges were smoother for this iteration. Figure 12 shows a cross section of the final shape of the lift arm. The mesh information was saved to a MSC/NASTRAN data file for this design. When attempting to solve this mesh, high aspect ratios in some of the elements were found again. 


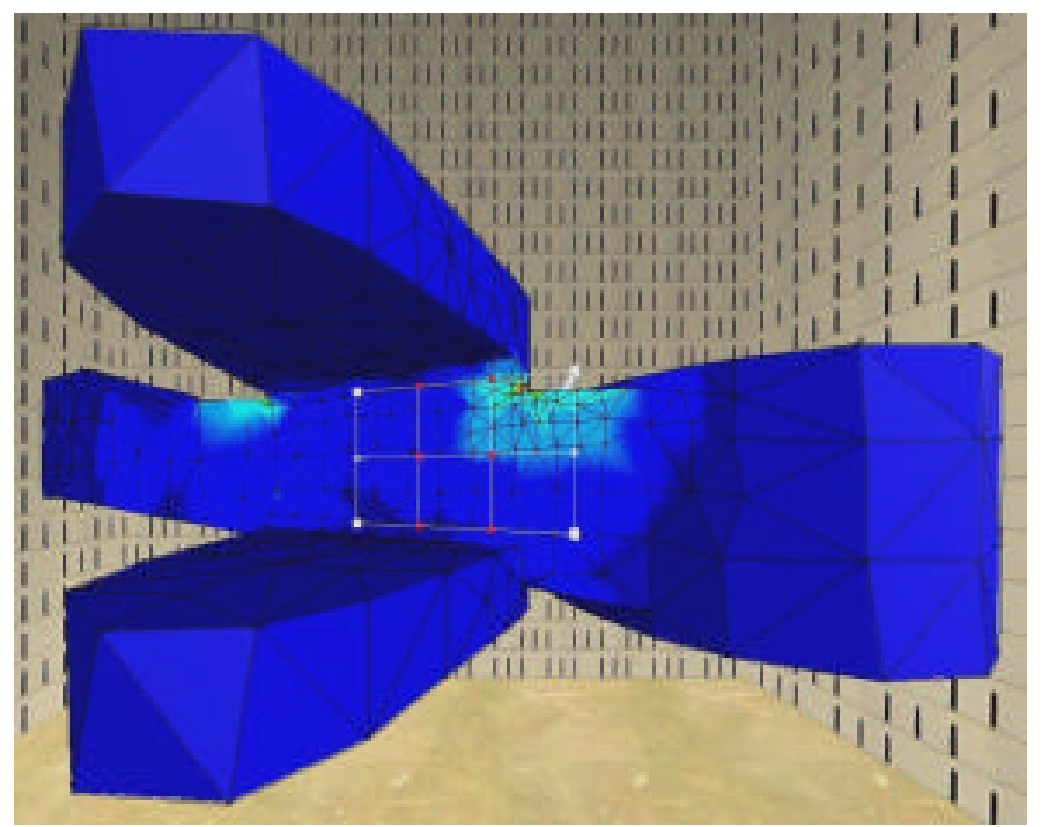

Figure 11. Design area and design variable for the final solution

Because of the complexity of the shape of the lift arm and the location of the high stress area, it was decided that it would be difficult to find a solution with the interactive stress analysis program that did not contain highly distorted elements. A comparison of the maximum vonMises stress approximations from the interactive stress analysis program showed that in the final design the highest stress was $418 \mathrm{MPa}$ which is a $42.6 \%$ reduction from the original maximum stress of $728 \mathrm{MPa}$. The approximate maximum stress of the new design was less than the yield strength of the material which is 440 MPa.

After the shape of the part was modified to produce this reduction in stresses, the new part shape was checked for collisions with the lift cylinder rod to verify the quality of the new part shape. The RAPID collision detection algorithm was used to check for collisions through the full range of motion of the lift arm. The two parts were checked at one degree increments from the down position $\left(0^{\circ}\right)$ to the up position $\left(37^{\circ}\right)$, and no

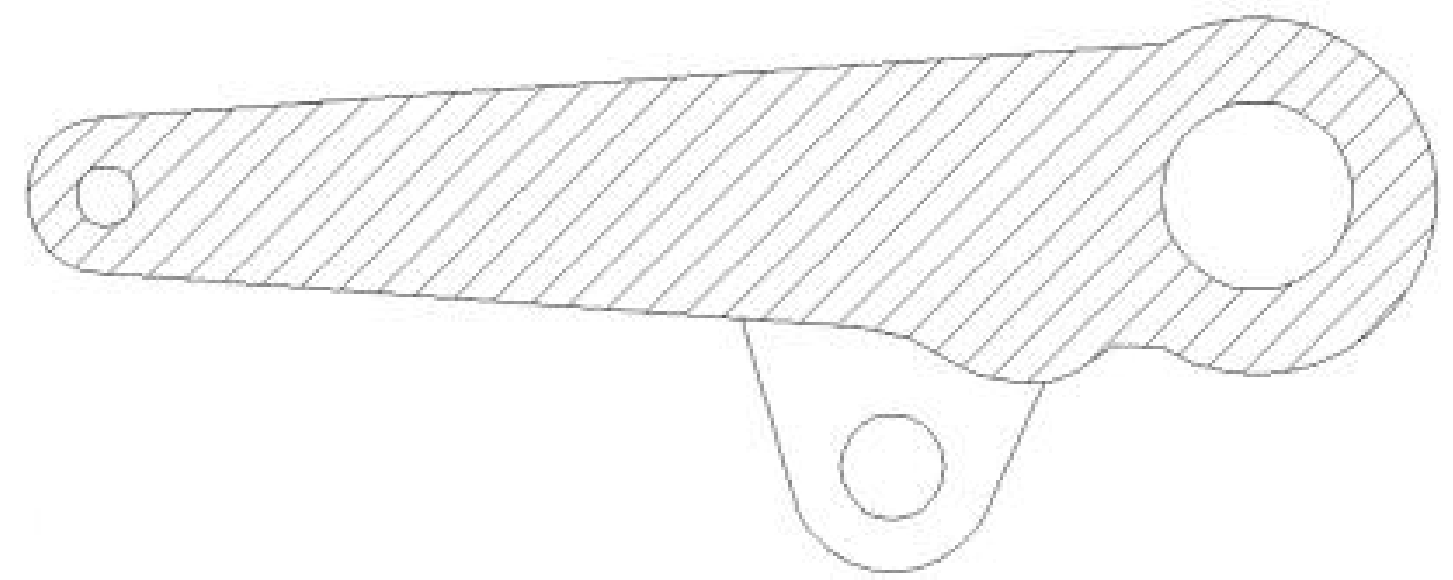

Figure 12. Final shape of the lift arm 
collisions were found. Figure 13 shows the lift arm and cylinder rod after checking for collisions.

To verify the maximum stress value found by the interactive stress analysis program, a new model of the lift arm's shape was created using Pro/Engineer based on the results of the interactive stress modification. This model was meshed and analyzed using MSC/NASTRAN. Results showed the maximum vonMises stress in the reanalyzed part was $466 \mathrm{MPa}$. This is $11.5 \%$ higher than what was indicated while finding the solution using the interactive program, this is attributed to the linear interpolation of the sensitivities performed in the program. This value is higher than the yield strength of the material, therefore more modification would be required to reduce the stresses in this part to an acceptable level.

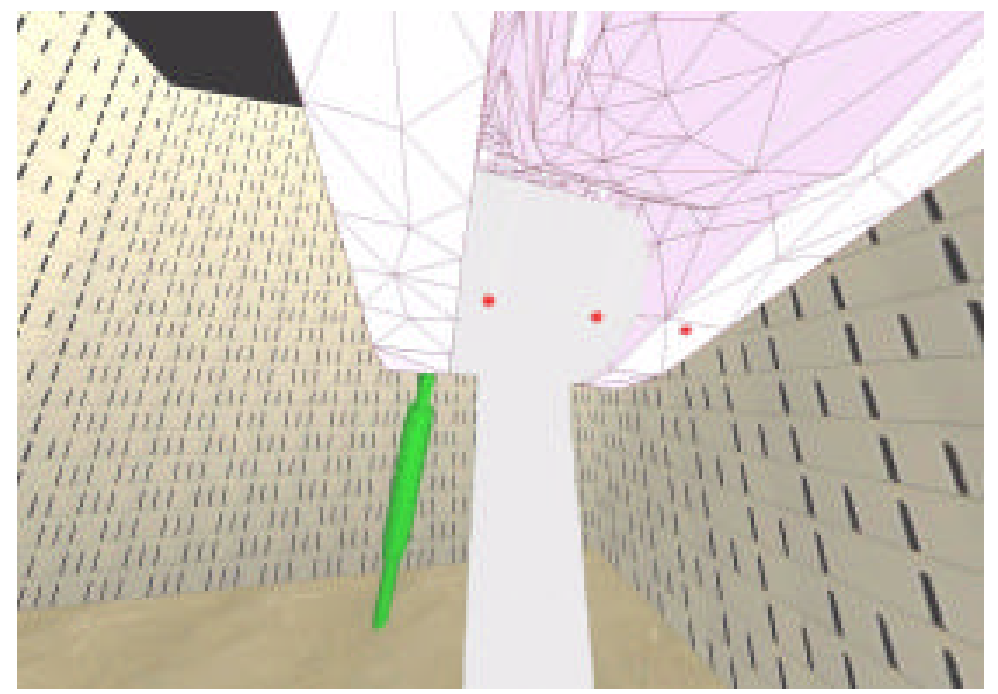

Figure 13. Collision checking of the final solution

\section{Discussion}

The first approach where the design area was selected to closely encompass the area of high stresses did reduce the maximum stress, however, this resulted in large shape changes in the design area which were unacceptable. After several trials of this nature failed to find an acceptable design, changing the shape of the lower part of the arm was investigated. This approach was not immediately obvious and therefore not the first approach. However, after the results were obtained, it was reasoned that, increasing the thickness of the lower part of the arm increases the mass moment of inertia of the arm and therefore reduces the stresses. The interactive stress analysis program allowed solution iterations for the fillet area as well as the area under the arm to be performed easily to find that shape modification of the lower part of the arm would reduce the stress in the fillet area.

The solution found using the interactive stress analysis program would be difficult to find using traditional methods. It was possible to create the new shape of the lift arm using Pro/Engineer, however it would have been difficult to iterate to that shape using traditional methods. Also, because of the potential collision with the lift cylinder rod in this area, many iterations would likely have been required to create this shape. 
Using a surround screen virtual environment for this example problem gave the user the ability to use natural movements to interact with the model. Defining the design area, selecting and changing the design variable, and examining the result were facilitated by viewing the model in three-dimensional stereo and interacting with the model using natural head and hand movements.

\section{Conclusions}

This research was successful in examining the challenges of applying virtual reality techniques to the interactive stress analysis of a tractor lift arm. The research presented here has extended previous work by implementing a surround screen virtual environment for displaying and interacting with the virtual geometry. Surrounding geometry and collision detection have also provided more information about the quality of the design to the user. This research combined NURBS-based free form deformation, finite element analysis, sensitivity analysis, collision detection and surround screen virtual reality techniques to facility structural shape design. The John Deere 8000 Series tractor lift arm was analyzed with these methods to evaluate the method's effectiveness when used on an industrial problem. MSC/NASTRAN provided the sensitivity information for the lift arm that made it possible to perform the interactive stress analysis in real-time.

The interactive stress analysis program found a solution to the example problem that would have been difficult to find through traditional methods, and was successful in reducing the stresses in the lift arm. Also, the surround screen virtual environment provided natural interaction with the lift arm model. In addition to these conclusions, this research also improved previous work in this area in the following ways:

1. Adding surrounding geometry and collision detection provides the designer with feedback on the quality of the design. These additions to previous methods indicate to the designer whether or not the new shape of the part interferes with the geometry surrounding the analysis part.

2. Evaluating the design in a three-dimensional immersive surround screen virtual environment gives the designer a better understanding of how the analysis part is changed and how it interfaces with surrounding geometry. It also has the potential to facilitate collaborative work with others interested in the part design.

\section{Acknowledgements}

This research was funded by Deere and Company. Facilities were provided by the Iowa Center for Emerging Manufacturing Technology. The input and guidance of Dr. Tsung-Pin Yeh, Engineering Animation, Inc. is gratefully acknowledged.

\section{References}

[1] Dai, F., Felger, W., Frühauf, T., Göbel, M., Reiners, D., and Zachmann, G., "Virtual Prototyping Examples for Automotive Industries," Virtual Reality World '96 Proceedings, February 1996.

[2] Deitz, D., "Real Engineering in a Virtual World," Mechanical Engineering, Vol. 117, pp. 78-85, 1995. 
[3] Gottschalk, S., Lin, M. C., and Manocha, D., "OBBTree: A Hierarchical Structure for Rapid Interference Detection," Proceedings of ACM SIGGRAPH, pp. 171-180, Aug. 1996.

[4] Kobe, G., "Virtual Interiors," Automotive Industries, Vol. 175, No. 5, pp. 52-54, 1995.

[5] Kurowski, P., and Szabo, B., "How to find errors in finite-element models," Machine Design, Vol. 69, No. 18, pp. 93-98, Sept. 25, 1997.

[6] Mahoney, D. P., “Driving VR,” Computer Graphics World, Vol. 18, No. 5, pp. 2223, 1995.

[7] Mahoney, D. P., "VR Drives Chrysler's New Cars," Computer Graphics World, Vol. 20, No. 7, pp. 61-62, 1997.

[8] MSC/NASTRAN User's Manual Version 70, The MacNeal Schwendler Corporation, Los Angeles, CA, 1997.

[9] Pro/Engineer Fundamentals Release 18.0, Parametric Technologies Corporation, Waltham, MA, 1997.

[10] Schmitz, B., "Great Expectations: The Future of Virtual Design," Computer-Aided Engineering, Vol. 14, No. 10, pp. 68-72, Oct. 1995.

[11] SDRC I-deas Master Series 5 Student Guide, Structural Dynamics Research Corporation, Milford, OH, 1997.

[12] Yeh, T. P. and Vance, J. M., “Applying Virtual Reality Techniques to Sensitivity Based Structural Shape Design," 1998 ASME Design Engineering Technical Conference, CDROM, Sept. 1998, accepted for publication in the ASME Journal of Mechanical Design.

[13] Yeh, T. P. and Vance, J. M., "Interactive Design of Structural Systems in a Virtual Environment," Computer Modeling and Simulation in Engineering, in press.

[14] Zhai, S., Buxton, W, and Milgram P., "The 'Silk Cursor': Investigating Transparency for 3D Target Acquisition," Proceedings of the CHI'94 Conference on Human Factors in Computing Systems, pp. 459-464, April 1994. 